\title{
Convex Ultrasound Image Reconstruction with Log-Euclidean Priors
}

\author{
José Seabra, João Xavier and João Sanches
}

\begin{abstract}
Image reconstruction from noisy and incomplete observations is usually an ill-posed problem. A Bayesian framework may be adopted do deal with this such inverse task by well posing the reconstruction problem. In this approach, the ill poseness nature of the reconstruction is removed by minimizing a two-term energy function. The first term pushes the solution toward the data and the second regularizes the solution.

A Bayesian algorithm for ultrasound image reconstruction and de-noising is proposed where an edge preserving prior is used to reduce the smoothing effect at the transitions. The prior distribution is based on log-Euclidean potential functions that are particular suitable in reconstruction problems under the constraint of positivity, that is, when the unknowns to be estimated should be positive, which is the case, where the noisy observations are modeled by a Rayleigh distribution.

In this paper, the reconstruction procedure is formulated as the optimization of a convex function and a Newton method is adopted to obtain the minimizer. This strategy guarantees a convergence to the global minimum in a small number of iterations. Experimental results, using synthetic and real medical images are shown. The proposed method produces images where speckle noise is effectively suppressed and important clinical details (organ and tissue transitions) are preserved.
\end{abstract}

\section{INTRODUCTION}

Ultrasound imaging is widely used in clinical practice for diagnostic purposes because it is non-invasive, non-ionizing, not expensive and real time based. Therefore, ultrasound image processing is an active field of research in areas such as $2 \mathrm{D}$ and $3 \mathrm{D}$ reconstruction, despeckling and textural and morphological characterization of tissues and organs.

The ultrasound images usually present a low signal to noise ratio (SNR) and are corrupted by a type of multiplicative noise called speckle that accompanies all coherent imaging modalities. It appears when images are obtained by using coherent radiation and is the result of the constructive and destructive interference of the echoes scattered from heterogeneous tissues and organs [1].

The characteristic granular speckle pattern present in the ultrasound images is evident in Fig. 5 ( $1^{\text {st }}$ row, $1^{\text {st }}$ col. of each image set). Its reduction or even its removal, while keeping the transitions that represent the anatomical details throughout the image would be beneficial for automatic contour segmentation or tissue characterization.

Several statistical models are proposed in the literature to describe this type of multiplicative noise [2]. One of the most used in Ultrasound (US), LASER and Synthetic Aperture Radar (SAR) is the Rayleigh distribution [3].

Corresponding author:jseabra@isr.ist.utl.pt

Systems and Robotics Institute / Instituto Superior Técnico, 1049-001 Lisbon, Portugal

Partially supported by FCT, under ISR/IST plurianual funding (POSC program, FEDER).
The speckle noise appearing in the US images is particular severe and the traditional methods based on the classical Additive White Gaussian Noise (AWGN) paradigm are not appropriate. To deal with this type of noise several methods have been proposed for de-noising and reconstruction based on wavelets [4], anisotropic diffusion [5] and level sets. Two methods, recently proposed, have shown to be particularly effective to deal with speckle noise: Non-Local Means (NLM) [6] and Squeeze Box Filter (SBF) [7].

Bayesian framework has also been successfully used in several medical imaging modalities, namely, in ultrasound imaging [8]. In this approach the ill-poseness nature of the reconstruction/de-noising problem is circumvented by using a priori information about the unknown image to be estimated. The estimation is formulated as an optimization task where a two-term energy function is minimized. The first term pushes the solution toward the observations and the second regularizes the solution. This second term, called prior term, introduces a priori knowledge about the solution by removing ambiguities that arise when only the observations are taken into account. The prior usually smooths the solution by removing the noise corrupting the observations and filling the gaps of non observed regions. However a difficulty must be addressed. The smoothing effect, which is crucial to noise removal, should not distort too much the edges of the solution associated with the anatomical details that are important from a clinical point of view.

In this paper a Bayesian reconstruction/de-noising algorithm for ultrasound data is proposed where an edge preserving prior based on log-Euclidean potential functions [9] is used. The reconstruction procedure is formulated as the optimization of a convex function and a Newton method is adopted to obtain the minimizer [10]. This strategy guarantees a convergence to the global minimum in a small number of iterations. Tests using synthetic and real data are presented to illustrate the application of the algorithm.

The paper is organized as follows. Section II formulates the problem and section III describes the optimization step. Section IV presents several results using synthetic and real medical data and Section V concludes the paper.

\section{Problem Formulation}

Let $\mathbf{X}=\left\{x_{i, j}\right\}$ and $\mathbf{Y}=\left\{y_{i, j}\right\}$ be a $N \times M$ original noiseless image and a noisy version respectively. The speckle noise corrupting $\mathbf{Y}=\left\{y_{i, j}\right\}$ is described by a Rayleigh distribution,

$$
p\left(y_{i, j} \mid x_{i, j}\right)=\frac{y_{i, j}}{x_{i, j}} e^{-\frac{y_{i, j}^{2}}{2 x_{i, j}}} .
$$




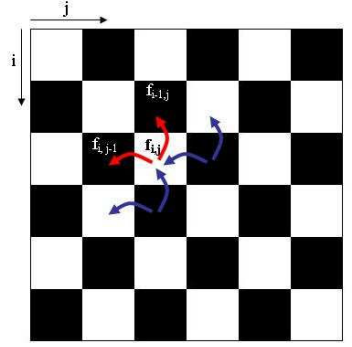

Fig. 1. 4-pixel causal neighborhood representation.

The estimation of $\mathbf{X}$ from $\mathbf{Y}$ is formulated as the following optimization task

$$
\hat{\mathbf{X}}=\arg \min _{\mathbf{X}} E(\mathbf{X}, \mathbf{Y})
$$

where $E(\mathbf{X}, \mathbf{Y})$ is an energy function.

The optimization problem, described by equation (2), is usually ill-posed in the Hadamard sense if $E(\mathbf{X}, \mathbf{Y})$ is the $\log$-likelihood function, $E(\mathbf{X}, \mathbf{Y})=\log p(\mathbf{Y} \mid \mathbf{X})$.

This difficulty may be overcome by using the maximum $a$ posteriori (MAP) criterion,

$$
E(\mathbf{X}, \mathbf{Y})=\underbrace{E_{Y}(\mathbf{X}, \mathbf{Y})}_{\text {data fidelity term }}+\underbrace{E_{X}(\mathbf{X})}_{\text {prior term }}
$$

where $E_{Y}(\mathbf{X}, \mathbf{Y})$, called data fidelity term, is the symmetric of the log-likelihood function

$$
E_{Y}(\mathbf{X}, \mathbf{Y})=-\log \left[\prod_{i, j=1}^{N, M} p\left(y_{i, j} \mid x_{i, j}\right)\right]
$$

where it is assumed statistical independence of the observations [11].

The prior term is used to regularize the solution by introducing a priori information about the image $\mathbf{X}$ to be estimated. Typically, the prior term is obtained by assuming that $\mathbf{X}$ is a Markov random field (MRF) where a neighboring system, $\mathscr{S}$, is considered to define spatial interactions among neighboring pixels (Fig. 1). By using the Emmerson-Clifford theorem the assumption that $\mathbf{X}$ is a MRF means that $p(\mathbf{X})$ is a Gibbs distribution

$$
p(\mathbf{X})=\frac{1}{Z} e^{-\alpha U(\mathbf{X})},
$$

where $Z$ is the partition function [12], $\alpha$ controls the prior strength and $U(\mathbf{X})$ is the Gibbs energy.

This Gibbs energy is usually defined as follows

$$
U(\mathbf{X})=\sum_{i, j} \rho\left(\mathbf{X}_{i, j}\right),
$$

where $\rho()$ is called potential function and $\mathbf{X}_{i, j}$ is a set of pixels containing the pixel $x_{i, j}$ and its neighbors.

A typical potential function is the quadratic one, $\rho\left(\mathbf{X}_{i, j}\right)=$ $\left(x_{i, j}-x_{i-1, j}\right)^{2}+\left(x_{i, j}-x_{i, j-1}\right)^{2}$, where neighboring pixel differences are quadratically penalized. This potential function leads to simple equations and is able to efficiently remove the noise. However, it also oversmooths the transitions, attenuating or removing important anatomical details.

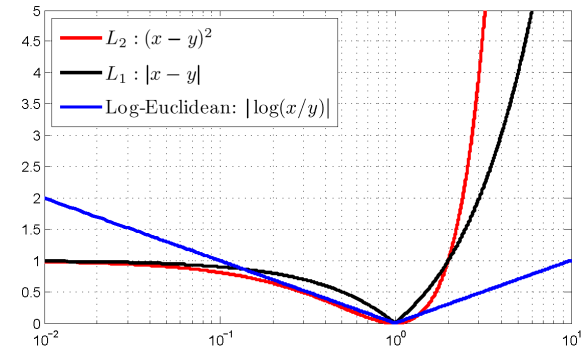

Fig. 2. Log-Euclidean gradient magnitude (blue) and the quadratic (red) and linear (black) potential functions.

To overcome this undesirable behavior, in this paper, an edge preserving [13] potential function is used,

$$
\rho\left(\mathbf{X}_{i, j}\right)=g_{i, j}
$$

where $g$ is the $\log$-Euclidean gradient magnitude at $(i, j)^{\text {th }}$ pixel,

$$
g(i, j)=\sqrt{\log ^{2}\left(x_{i, j} / x_{i-1, j}\right)+\log ^{2}\left(x_{i, j} / x_{i, j-1}\right)}
$$

This potential function is appropriated when positivity constraint is imposed at the solution, $x_{i, j}>0$, which is the case. Fig. 2 shows this potential function, $\rho(x, y)=\log ^{2}(x / y)$, for $0 \leq x \leq 10$ and $y=1$. The log-Euclidean [9] potential function penalizes much more small differences between neighboring pixels, when they are small than the quadric potential function. On the contrary, if the difference is large, which usually happens at the transitions, the log-Euclidean potential function penalizes less than the quadratic prior, smoothing less the transitions.

The log-Euclidean prior is based on the distance function $\rho(x, y)=|\log (x / y)|$ that is in fact a metric because the following conditions hold:

1) $\rho(x, y) \geq 0$

2) $\rho(x, y)=0$ if and only if $x=y$

3) $\rho(x, y)=\rho(y, x)$

4) $\rho(x, z)+\rho(z, y) \geq \rho(x, y)$.

\section{CONVEX Optimization}

The energy function to be minimized is given by

$$
E(\mathbf{X}, \mathbf{Y})=\sum_{i, j}\left[\frac{y_{i, j}^{2}}{2 x_{i, j}}+\log \left(x_{i, j}\right)\right]+\alpha \sum_{i, j} g_{i, j}
$$

where $g_{i, j}$ is defined in (8).

This energy function is not convex because $\log (x)$ is concave and $1 / x$ and $\rho(x)$ are convex. Therefore, let us consider the following variable change $f=\log (x)$ (notice that $\log (x)$ is a monotonic function). The new energy function to minimize is

$$
\begin{aligned}
E(\mathbf{F}, \mathbf{Y}) & =\sum_{i, j}\left[\frac{y_{i, j}^{2}}{2} e^{-f_{i, j}}+f_{i, j}\right] \\
& +\alpha \sum_{i, j} \sqrt{\left(f_{i, j}-f_{i-1, j}\right)^{2}+\left(f_{i, j}-f_{i, j-1}\right)^{2}+\varepsilon}
\end{aligned}
$$


which is now convex, because, $e^{-f}$ and $\rho(f)$ are both convex.

The minimization of the energy function (10) is iteratively performed by using a line search [10] algorithm in which the basic step is $x_{k+1}=x_{k}+\alpha_{k} d_{k}$, with $\alpha_{k}>0$. A continuous variation strategy (here termed cooling) is used where a small decreasing constant $\varepsilon$, updated at each iteration, is added in order to deal with the non-smooth term of (10). Different strategies to compute the descent direction $d_{k}$ were tested:

1) Barzilai-Borwein steepest (gradient) method, $d_{k}=$ $-\nabla E\left(x_{k}\right) / \alpha_{k}$, with $\alpha_{k}=\frac{d_{k-1}^{T} y_{k-1}}{d_{k-1}^{T} d_{k-1}}$ and $\frac{y_{k-1}^{T} y_{k-1}}{y_{k-1}^{T} d_{k}-1} \cdot \varepsilon=$ $10^{-6}$

2) Barzilai-Borwein steepest (gradient) method, with continuous variation of: $\varepsilon=1 \rightarrow 0$;

3) Newton algorithm, with continuous variation of $\varepsilon=$ $1 \rightarrow 0$, where $d_{k}=-(\underbrace{\nabla^{2} E\left(x_{k}\right)}_{\text {Hessian }^{1}})^{-1} \nabla E\left(x_{k}\right)$.

The main steps of the overall convex reconstruction/denoising algoorithm for ultrasound images are listed in the following table,

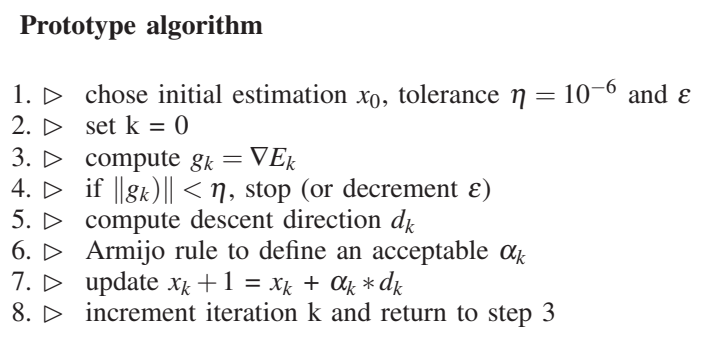

The final solution is $\hat{\mathbf{X}}=e^{Z}$. As it is shown in Fig. 3 the Newton method performs better than the other descent approaches used in the optimization algorithm, which is an expected behavior because the search direction takes into account not only the gradient of the energy function but also its second-order information. The Newton algorithm seems to be very computationally demanding because the Newton direction is obtained by solving a rather large linear system at each iteration. However, we are dealing with highly sparse Hessian matrices (a variable is coupled only with its spatial neighbors) which makes the method feasible.

\section{Results}

In this section we present three examples of reconstruction using synthetic and real data. In the synthetic case we use both 1D and 2D data corrupted with multiplicative Rayleigh noise. In the real case, several ultrasound images are presented.

\section{A. Synthetic data - $1 D$ and $2 D$}

In this experiment we have first generated a vector $\mathrm{X}$ with dimension $N=1024$ corresponding to a rectangular shaped function with $X_{\max }=5000$ and $X_{\min }=500$. A

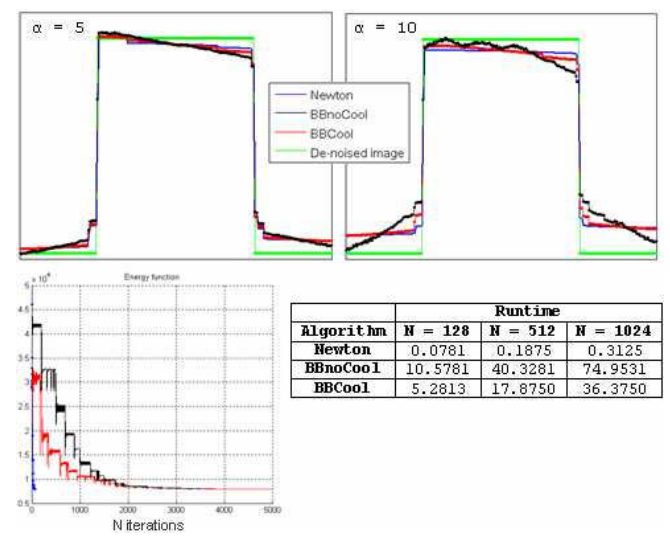

Fig. 3. Profiles of the reconstructed data. Performances of the different descent methods used.

Rayleigh distributed vector $\mathrm{Y}$ with parameter $\mathrm{X}$ was then generated. Results of de-noising and performance using the three different descent strategies are depicted in Fig. 3. Fig. 3 (bottom) illustrates the outstanding performance of the Newton algorithm when compared with the other methods. Therefore, the Newton method is used from now on.

Moreover, it is shown that lower values for the parameter $\alpha$ (prior strength) lead to sharper solutions were the transitions are better preserved. This parameter is manually tuned and was selected in a trial and error basis.

In the second example, synthetic images (a square and a set of different-sized ellipses) corrupted with Rayleigh noise were generated (see Fig. 4(a-b) $\left(1^{s t}\right.$ row, $1^{s t}$ col.)). The distribution parameters were $X_{\max }=5000$ and $X_{\min }=1000$. Reconstruction results obtained with the proposed method (Fig. 4(a-b) $\left(1^{\text {st }}\right.$ row, $2^{\text {nd }}$ col.)) were visually compared with SBF (Fig. 4(a-b) (2 ${ }^{\text {nd }}$ row, $1^{\text {st }}$ col.)) and NLM (Fig. $4(\mathrm{a}-\mathrm{b})\left(2^{\text {nd }}\right.$ row, $2^{\text {nd }}$ col. $\left.)\right)$ algorithms. It is observed that the proposed algorithm provides images where the speckle noise is successfully attenuated while the edges are better preserved. This observation is supported by inspecting the de-noised image profiles (Fig. 4 (bottom)). To allow a more objective comparison the signal to noise ratio (SNR) was also computed. For both images, the best SNR is achieved with the method proposed in this paper.

\section{B. Medical data}

In the last example, application of the proposed reconstruction algorithm and comparison with SBF and NLM was done in real medical data. 4 different ultrasound images are presented ( $1^{s t}$ row, $1^{\text {st }}$ col. of each set of images) and reconstruction results using the proposed method (RaylCx $1^{\text {st }}$ row, $2^{\text {nd }} \mathrm{col}$.), SBF (2 $2^{\text {nd }}$ row, $1^{\text {st }} \mathrm{col}$.) and NLM ( $2^{\text {nd }}$ row, $2^{\text {nd }}$ col.) are depicted in Fig. 5. At the bottom of each set of images, profiles from the de-noised images using the different reconstruction algorithms are shown. Regularization parameters used by the three algorithms were kept constant along the different images to study the robustness of the denoising methods.

By qualitative inspection of the images and corresponding 

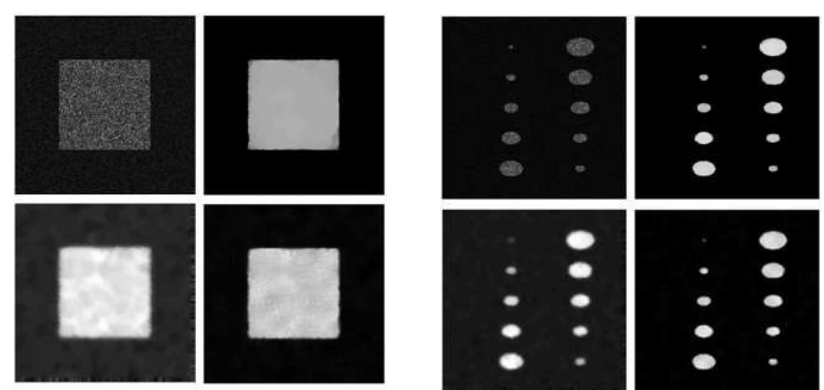

a)

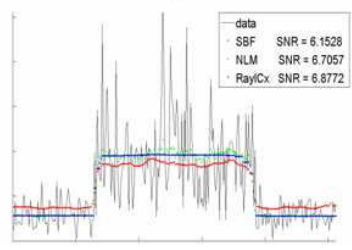

b)
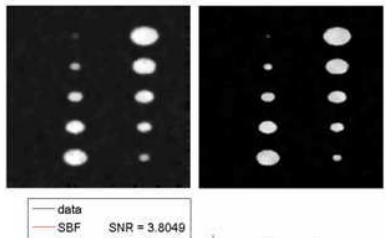

NLM SNR $=6.5693$

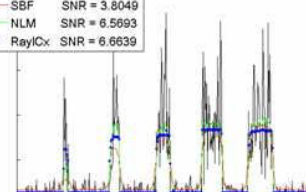

Fig. 4. De-noising results of two artificially generated noisy images: a noisy blank square (left) and a set of noisy different-sized blank ellipses (right), using the proposed method and two other de-noising algorithms. (bottom) De-noised image profiles.

profiles, the proposed de-noising algorithm, which is time competitive with the others used, attains cleaner images with the relevant organ edges being well preserved. For instance, Fig.5(a) shows that the SBF overestimates the original edges and Fig.5(d) shows that the heart chambers walls are more clearly defined using the RaylCx algorithm while the other two produce smoother images.

\section{CONCLUSIONS}

This paper proposes a Bayesian ultrasound image reconstruction/de-noising (despeckle) algorithm using the maximum a posteriori (MAP) criterion. The algorithm is formulated as the optimization of a convex energy function. The convexity of the function and the minimization by using the Newton method guarantee a continuous convergence to the global minimum in a small number of iterations.

The prior distribution, used to regularize the solution and well pose the intrinsic ill-posed maximum likelihood solution, is a Gibbs distribution with potential functions based on the Log-Euclidean gradient magnitude of the de-noised image. The Log-Euclidean gradient magnitude is based on the LogEuclidean distance metrics that are particular suitable when the optimization is performed in $R_{+}^{N}$, which is the case. In fact, the speckle noise, corrupting the ultrasound images is modeled by a Rayleigh distribution where the parameters to be estimated are positive.

Tests using synthetic and real data illustrate the application of the algorithm have show the effectiveness of the proposed method. Furthermore, comparison with two state of the art speckle algorithms described in the literature shows that the proposed method outperforms the other methods.

\section{REFERENCES}

[1] J. Abbot and F. Thurstone, "Acoustic speckle: Theory and experimental analysis," Ultrasound Imaging, vol. 1, pp. 303-324, 1979.

[2] O. V. Michailovich and A. Tannenbaum, "Despeckling of medical ultrasound images," IEEE Transactions on Ultrasonics, Ferroelectrics and Frequency Control, vol. 53, no. 1, pp. 64-78, 2006.

[3] C. Burckhardt, "Speckle in ultrasound b-mode scans," IEEE Transations on Sonics and Ultrasonics, vol. SU-25, no. 1, pp. 1-6, January 438 1978.
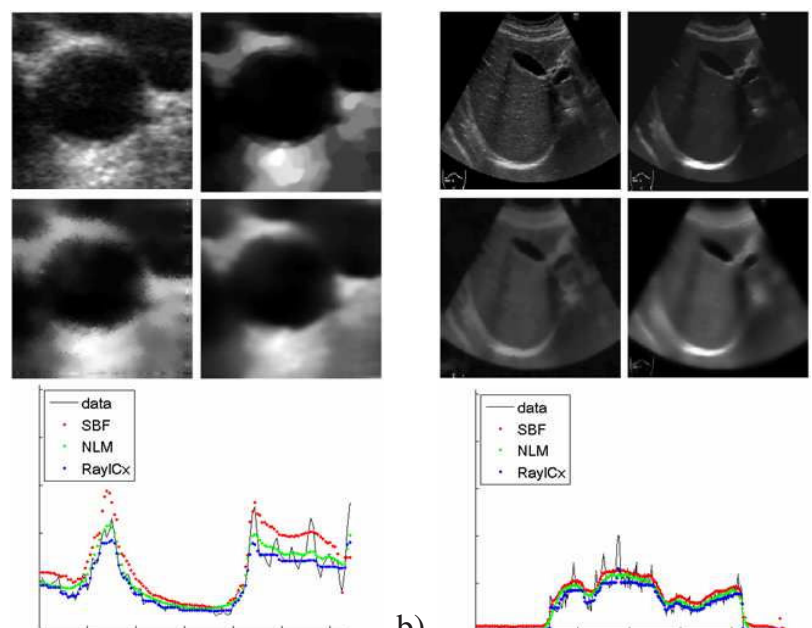

a)
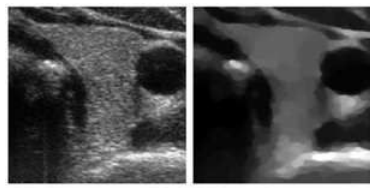

b)
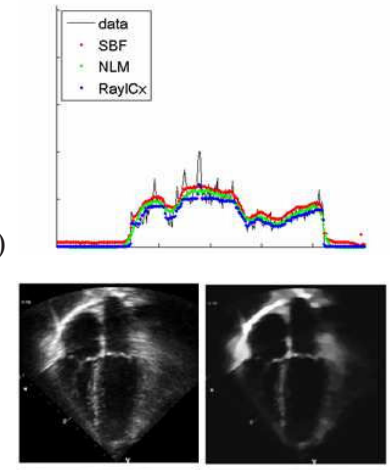

c)
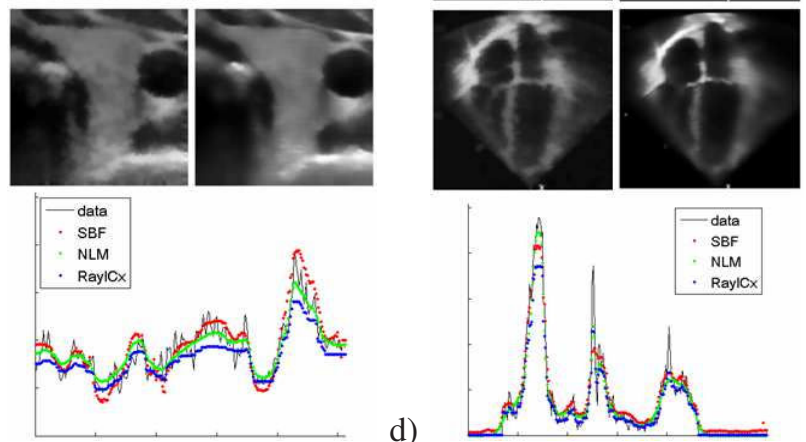

d)

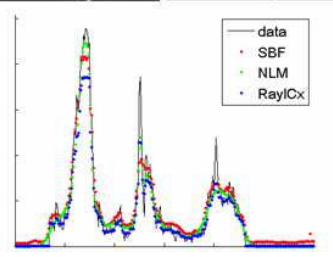

Fig. 5. De-noising results of ultrasound images of the carotid (a), liver(b), thyroid (c) and heart (d), using the proposed RaylCx and SBF and NLM methods. (a-d) (bottom) image profiles are also depicted.

[4] S. G. Chang, B. Yu, and M. Vetterli, "Adaptive wavelet thresholding for image denoising and compression," IEEE Transactions on Image Processing, vol. 9, no. 9, pp. 1532-1546, 2000.

[5] Y. Yu and S. Acton, "Speckle reducing anisotropic diffusion," 2002.

[6] A. Buades, B. Coll, and J. M. Morel, "A review of image denoising algorithms, with a new one," Multiscale Modeling and Simulation, vol. 4, no. 2, pp. 490-530, 2005.

[7] P. C. Tay, P. C. Tay, S. T. Acton, S. T. Acton, J. A. Hossack, and J. A. Hossack, "Ultrasound despeckling using an adaptive window stochastic approach ultrasound despeckling using an adaptive window stochastic approach," in Image Processing, 2006 IEEE International Conference on, 2006, pp. 2549-2552.

[8] M. Banham and A. Katsaggelos, "Digital image restoration," IEEE Signal Processing Magazine, vol. 14, no. 2, March 1997.

[9] Ayache, "Anisotropic filtering for model-based segmentation of $4 \mathrm{~d}$ cylindrical echocardiographic images," Pattern Recognition Letters Special Issue on Ultrasonic Image Processing and Analysis, vol. 24, pp. 815-828, February 2003.

[10] S. Boyd and L. Vandenberghe, Convex Optimization. Cambridge University Press, March 2004.

[11] J. Dias, T. Silva, and J. Leitão, "Adaptive restoration of speckled SAR images using a compound random markov field," in Procedings IEEE International Conference on Image Processing, Vol.II. Chicago, USA: IEEE, October 1998, pp. 79-83.

[12] S. Geman and D. Geman, "Stochastic relaxation, gibbs distributions and the bayesian restoration of images," IEEE Transactions on Pattern Analysis and Machine Intelligence, vol. 6, no. 6, pp. 721-741, November 1984.

[13] J. Sanches, J. B. Dias, and J. Marques, "Minimum total variation in 3d ultrasound reconstruction," IEEE International Conference on Image Processing - ICIP 2005, September 2005. 